Bangladesh J. Plant Taxon. 14(2): 147-162, 2007 (December)

\title{
AN ANNOTATED CHECKLIST OF LAURACEAE IN BANGLADESH
}

\author{
Hosne Ara ${ }^{1}$, Md. Manzur-ul-Kadir Mia And Bushra Khan \\ Bangladesh National Herbarium, Chiriakhana Road, Mirpur 1 \\ Dhaka 1216, Bangladesh
}

Key words: Checklist, Lauraceae, Bangladesh

\begin{abstract}
A preliminary report on the species of the Laurel family in Bangladesh is presented in this paper. In Bangladesh, the family is represented by 13 genera and 46 species. Updated nomenclature with synonyms, local and English names, flowering and fruiting time, diagnostic characteristics, geographical distribution and occurrence within Bangladesh have been provided under each taxon.
\end{abstract}

\section{Introduction}

The family Lauraceae is represented by 32 genera and 2,500 species distributed mostly in the tropical and subtropical regions of the world (Heywood 1993). A comprehensive and systematic analysis on the availability and distribution of Lauraceae in Bangladesh has not been prepared yet. Hooker $(1886,1890)$ reported 15 genera and 204 species for British India, out of which 11 genera and 39 species were recorded from the present Bangladesh territory. Prain (1903) recorded 23 species and 9 genera from the area of present Bangladesh, whereas Heinig (1925) listed 20 species distributed under 9 genera from the Chittagong and Chittagong Hill Tract region. Sinclair (1955) enumerated 5 species and 3 genera from the area of Cox's Bazar. Alam (1988) recorded 30 species and 9 genera from the forests of Sylhet region.

The present taxonomic enumeration of the family is based mostly on the herbarium materials preserved in the Bangladesh National Herbarium (DACB) and Dhaka University Herbarium (DUH), and available literature by different workers, who worked on the flora of this region, viz. Cowan and Cowan (1929), Raizada (1941), Huq and Khan (1984), Khan et al. (1994), Mia and Khan (1995), Rahman and Hassan (1995), Rahman and Uddin (1997), Uddin et al. (1998), Uddin and Rahman (1999), Rashid et al. (2000), Khan and Huq (2001) and Rahman (2004a, b).

This checklist contains the names of 46 species and 13 genera of Lauraceae so far reported from the territory of Bangladesh. The following literature have been consulted for the up-to-date nomenclature, viz. Kostermans (1978), Farr et al. (1979) and Hara et al. (1982). The genera and species have been arranged in alphabetical order and each species is accompanied by its local names, salient features, flowering ( $F l$.) and fruiting (Fr.) time, occurrence in Bangladesh and geographic distribution.

\footnotetext{
${ }^{1}$ Corresponding author. E-mail : bnh_mirpur@yahoo.com
} 


\section{Taxonomic enumeration}

Actinodaphne C.G.D. Nees in Wall., Pl. As. Rar. 2: 61-68 (1831).

1. Actinodaphne angustifolia Nees in Wall., Pl. As. Rar. 3: 31 (1832). Hook. f., Fl. Brit. Ind. 5: 152 (1886); Prain, Beng. Pl. 2: 674 (1903-reprint 1963); Heinig, List Chittagong: 56 (1925). A. hookeri Meissn., DC. Prodr. 15 (1): 218 (1864); Hook. f., Fl. Brit. Ind. 5: 149 (1886).

Local name: Modanmosta.

A medium to large-sized evergreen tree, up to $20 \mathrm{~m}$ high. Leaves in whorls, very variable in length and breadth, $10-25 \times 3.5-6.2 \mathrm{~cm}$, oblanceolate, lanceolate or elliptic. Inflorescence scattered, umbellate. Fruit globose, up to $0.8 \mathrm{~cm}$ long. Fl. \& Fr.: JuneDecember.

Occurrence in Bangladesh: Chittagong, Chittagong Hill Tracts and Sylhet forests (Hooker 1886, Heinig 1925).

Distribution: Indo-Malaya and North-East India.

2. Actinodaphne obovata (Nees) Blume, Mas. Bot. 1: 342 (1851). Hook. f., Fl. Brit. Ind. 5: 153 (1886). Tetradenia obovata Nees in Wall., Pl. As. Rar. 2: 64 (1831).

Local name: Kolapata (Sylhet).

A small to medium-sized tree. Leaves $17.5-45.0 \times 6.2-15.0 \mathrm{~cm}$, very variable in size and shape, obovate or elliptic-oblong. Fruit ellipsoid, more than $2 \mathrm{~cm}$ long. Fl. \& Fr.: March-August.

Occurrence in Bangladesh: Sylhet (Alam 1988).

Distribution: Eastern Himalaya and Sub-himalayan regions (Assam, Khasia hills, Meghalaya, Monipur and Sikkim).

Beilschmiedia Nees in Wall., Pl. As. Rar. 2: 69 (1831).

3. Beilschmiedia assamica Meissn. in DC. Prodr. 15 (1): 64 (1864). Hook. f., Fl. Brit. Ind. 5: 124 (1886); Brandis, Ind. Trees: 529 (1906); Kanjilal et al., Fl. Assam 4: 53 (1940-reprint 1982).

Local name: Naga-sutrong (Sylhet).

A large tree, 15-25 m high. Leaves 6-20 × 3-8 cm, opposite or sub-opposite, elliptic, oblong-elliptic or lanceolate. Inflorescence paniculate, panicles 8-16 cm long. Fruit 2.5$5.0 \times 1.5-2.0 \mathrm{~cm}$, ellipsoid or ovoid-oblong. Fl. \& Fr.: December-April.

Occurrence in Bangladesh: Sylhet (Alam 1988).

Distribution: Myanmar and North-East India (Assam). 
4. Beilschmiedia fagifolia Nees in Wall., As. Rar. 2: 69 (1831). Hook. f., Fl. Brit. Ind. 5: 122 (1886); Kanjilal et al., Fl. Assam 4: 52 (1940-reprint 1982).

A small to medium-sized tree, up to $10 \mathrm{~m}$ high. Leaves alternate or sub-opposite, 5$12 \times 2-5 \mathrm{~cm}$, elliptic, oblong-elliptic, or elliptic-lanceolate. Inflorescence short, crowded panicles. Fruit elliptic-oblong, c 0.5 cm long. Fl. \& Fr.: June-December.

Occurrence in Bangladesh: Sylhet (Hooker 1886, Alam 1988).

Distribution: North-East India.

5. Beilschmiedia gammieana King ex Hook. f., Fl., Brit. Ind. 5: 124 (1886).

A small to medium-sized tree with spreading branches. Leaves opposite or subopposite, $15-30 \times 5-8 \mathrm{~cm}$, oblong-lanceolate or elliptic-oblong. Inflorescence axillary panicles or terminal racemes, very short, up to $3 \mathrm{~cm}$ long. Fruit a berry or drupe, oblong, on thickened pedicel, 1.8-3.0 $\times$ 0.8-1.8 cm, globose-obovoid, shortly apiculate. Fl. \& Fr.: March-November.

Occurrence in Bangladesh: Greater Sylhet (Alam 1988).

Distribution: Bhutan, India and Nepal.

6. Beilschmiedia roxburghiana Nees in Wall., Pl. As. Rar. 2: 69 (1831). Hook. f., Fl. Brit. Ind. 5: 121 (1886); Prain, Beng. Pl. 2: 672 (1903-reprint 1963). Laurus bilocularis Roxb., Fl. Ind. 2: 311 (1824).

Local name: Serai-guti (Sylhet).

A small to medium-sized tree, up to $10 \mathrm{~m}$ high with a compact, oval crown branches from the base. Leaves 10-25 $\times$ 4-10 cm, alternate, oblong, elliptic-lanceolate or elliptic oblong. Inflorescence peduncled panicles or racemes, 3-6 cm long, few-flowered, axillary or from axils of the fallen leaves or lateral from the axils of the caducous scales. Fruit baccate, obovoid-oblong, 2-3 cm long, dark purple when ripe. Fl. \& Fr.: March-August.

Occurrence in Bangladesh: Sylhet forest areas (Alam 1988) and Dhaka (Brandis 1906).

Distribution: North-Eastern India and Myanmar.

$$
\text { Cassytha L., Sp. Pl.: } 35 \text { (1753). }
$$

7. Cassytha filiformis L., Sp. Pl.: 35 (1753). Hook. f., Fl. Brit. Ind. 5: 188 (1886); Prain, Beng. Pl. 2: 676 (1903-reprint 1963); Heinig, List Chittagong: 57 (1925); Sinclair, Bull. Bot. Soc. Beng. 9 (2): 105 (1955).

Local name: Akashbel.

A herbaceous twining parasite, attaching itself by haustoria to the host plant forming a web of leafless cords over bushes. Stem dark-green, puberulous when young, glabrescent with age. Leaves none or reduced to a few minute hyaline scales. Fruit 
globose, c $0.7 \mathrm{~cm}$ in diameter, white, smooth. Fl. \& Fr.: Almost throughout the year, usually July-October.

Occurrence in Bangladesh: Coastal districts and Islands (Sinclair 1955).

Distribution: India, Sri Lanka, Tropical East Asia, Africa, Australia and America.

Cinnamomum Schaeffer, Bot. Exped.: 74 (1760).

8. Cinnamomum bejolghota (Buch.-Ham.) Sweet, Hort. Brit. ed. 1: 344 (1827). Laurus bejolghota Buch.-Ham. in Trans. Linn. Soc. 13: 559 (1822). Cinnamomum obtusifolium Roxb. ex Nees in Wall., Pl. As. Rar. 2: 73 (1831); Hook. f., Fl. Brit. Ind. 5: 128 (1886); Prain, Beng. Pl. 2: 673 (1903-reprint 1963); Heinig, List Chittagong: 56 (1925); Sinclair, Bull. Bot. Soc. Beng. 9 (2): 105 (1955).

Local names: Tezpat, Ram tejpat, Kinton (Chittagong, Chittagong Hill Tracts).

A large tree, up to $25 \mathrm{~m}$ high. Leaves opposite or sub-opposite, 15-30 $\times 4-9 \mathrm{~cm}$, elliptic-oblong or elliptic. Inflorescence panicles, panicles usually longer than the leaves. Fruits 0.6-1.2 cm long, ellipsoid or sub-globose. Fl. \& Fr.: January-July.

Occurrence in Bangladesh: Chittagong, Chittagong Hill Tracts and Sylhet regions (Hooker 1886, Heinig 1925).

Distribution: Central and Eastern Himalaya, Andaman Island and Myanmar.

9. Cinnamomum camphora (L.) J. Presl, Priroz. Rostlin 2: 36 \& 47-56 (1825). Hook. f., Fl. Brit. Ind. 5: 134 (1886); Prain, Beng. Pl. 2: 673 (1903-reprint 1963). Laurus camphora L., Sp. Pl. 369 (1753).

Local name: Karpur. English name: Camphor tree

A small to medium-sized tree with spreading crown. Leaves alternate or spirally arranged, ovate-elliptic to elliptic or sub-ovate-elliptic, 3-10 $\times 1-5 \mathrm{~cm}$. Inflorescence panicles, axillary, slender, many-flowered, up to $10 \mathrm{~cm}$ long with few short branches. Fruit globose, slightly fleshy, 5-10 mm in diameter. Fl. \& Fr.: March-July.

Occurrence in Bangladesh: Almost every where in Bangladesh (mostly planted).

Distribution: Native to China and Japan, widely cultivated all over the world.

10. Cinnamomum glanduliferum (Wall.) Meissner., DC. Prodr. 15 (1): 25 (1864). Hook. f., Fl. Brit. Ind. 5: 135 (1886). Laurus glandulifera Wall. in Tr. S. Med. Phys. Calc. 1: 45 \& 51, t. 1 (1825).

A large tree, up to $25 \mathrm{~m}$ high. Leaves alternate, very variable in size and shape, usually 4-12 × 3-6 cm, ovate or obovate-elliptic, orbicular or lanceolate. Inflorescence panicles, axillary or terminal, up to $8 \mathrm{~cm}$ long, few-flowered, appearing after or together with leaves. Fruit up to $2.5 \mathrm{~cm}$ long, obovoid. Fl. \& Fr.: April-August.

Occurrence in Bangladesh: Chittagong and Sylhet (Brandis 1874). 
Distribution: Central Himalaya and eastern India, Nepal and Sikkim.

11. Cinnamomum glaucescens (Nees) Meissn., DC. Prodr. 15 (1): 25 (1864). Cecicodaphne glaucescens Nees in Wall., Pl. As. Rar. 2: 70 (1831). C. cecidodaphne Meissn. in DC. Prodr. 15 (1): 25 (1864); Hook. f., Fl. Brit. Ind. 5: 135 (1886); Brandis, Ind. Trees: 534 (1906).

Local names: Gonoroi, Gonori (Sylhet).

A medium to large-sized tree with spreading crown. Leaves alternate, 5-10 $\times 2-5 \mathrm{~cm}$, elliptic-lanceolate or broadly elliptic. Inflorescence panicles, 4-6 cm long. Fruit 1.5-3.0 cm long, ellipsoid, oblong. Fl. \& Fr.: February-November.

Occurrence in Bangladesh: Greater Sylhet (Hooker 1886).

Distribution: Bhutan, India, Nepal and Sikkim.

12. Cinnamomum iners Reinw., Blume Bijdr.: 570 (1826). Hook. f., Fl. Brit. Ind. 5: 136 (1886); Heinig, List Chittagong: 56 (1925).

Local names: Tez-bohu, Kosturi, Karuyea (Chittagong, Chittagong Hill Tracts).

A medium-sized tree about $12 \mathrm{~m}$ tall. Leaves $12.5-18.0 \times 3.8-7.5 \mathrm{~cm}$, elliptic or elliptic-oblong. Inflorescence panicles, c $15 \mathrm{~cm}$ long, very lax-flowered. Fruit ellipsoid, black, pulpy, blent, 1.0-1.5 cm long. Fl. \& Fr.: January-February.

Occurrence in Bangladesh: Chittagong and Chittagong Hill Tracts (Heinig 1925).

Distribution: India, Myanmar, Malay Peninsula and Indonesia.

13. Cinnamomum nitidum Blume, Rumph. 1: 35 (1836). Brandis, Ind. Trees: 533 (1906). Laurus culitlaban Roxb. Hort. Beng. (30); Fl. Ind. II. 299 (1824). Cinnamomum encalyptoides Nees (1831).

Local name: Kabab.

A shrub or tree, with short appressed branches. Leaves 7-22 cm long, glabrous, elliptic-oblong or linear-oblong, obtuse, 3-nerved. Inflorescence panicles, slender, longpeduncled, often longer than the leaves. Fruit ellipsoid, 1.0-1.5 cm long. Fl. \& Fr.: Not on record.

Occurrence in Bangladesh: Mirpur National Botanic Garden, Dhaka (Dey 2006).

Distribution: Malay Islands, Molaccas and Myanmar.

14. Cinnamomum pauciflorum Nees in Wall., Pl. As. Rar. 2: 75 (1831). Hook. f., Fl. Brit. Ind. 5: 129 (1886); Brandis, Ind. Trees: 533 (1906); Kanjilal et al., Fl. Assam 4: 57 (1940-reprint 1982). Laurus recurvata Roxb., Fl. Ind. 2: 301 (1832).

A large shrub or a small tree. Leaves usually opposite, 5-12 $\times 2-5 \mathrm{~cm}$, elliptic-ovate, ovate-lanceolate to oblong-lanceolate. Inflorescence axillary panicles, up to $8 \mathrm{~cm}$ long, few-flowered. Fruits 0.7-1.0 cm across, globose or ellipsoid. Fl. \& Fr.: April-November. 
Occurrence in Bangladesh: Sylhet (Hooker 1886).

Distribution: North-east India.

15. Cinnamomum tamala (Buch.-Ham.) Nees \& Eberm., Handb. Med.-Pharm. B. 2: 426 (1831). Hook. f., Fl. Brit. Ind. 5: 128 (1886); Prain, Beng. Pl. 2: 673 (1903reprint 1963); Datta \& Mitra, Bull. Bot. Soc. Beng. 7 (1\&2): 12 (1953). Laurus tamala Buch.-Ham. in Tr. Linn. S. 13: 555 (1822).

Local names: Huara, Tejpata. $\quad$ English names: Cassia cinnamon, Cassia lignea.

A medium-sized evergreen tree, up to $15 \mathrm{~m}$ high. Leaves opposite or sub-opposite, 10-15 × 2.5-6.2 cm, ovate-oblong or elliptic to oblong-lanceolate. Inflorescence panicles, panicles as long as leaves. Fruit a drupe, c $1 \mathrm{~cm}$ long, black when ripe, ovoid or globose. Fl. \& Fr.: February-October.

Occurrence in Bangladesh: Hilly forest areas of greater Sylhet (Alam 1988).

Distribution: Tropical and sub-tropical Himalayan regions, Bhutan, India and Nepal.

16. Cinnamomum verum J. Prest, Prin. Rostlin 2: 36 (1825). Cinnamomum zeylanicum Blume Bijdr. xi.: 568 (1826); Nees in Wall., Pl. As. Rar. 2: 74 (1831).

Local names: Darchini, Daruchini, Dalchini. English names: Cinnamon, True cinnamon.

A medium-sized evergreen tree, all parts glabrous. Leaves opposite or sub-opposite, rarely alternate, ovate, ovate-lanceolate. Inflorescence lax panicles, terminal, manyflowered, usually longer than leaves. Fruit 1.3-1.7 cm long, oblong or ovoid-oblong, minutely apiculate. Fl. \& Fr.: January-March.

Occurrence in Bangladesh: Chittagong Hill Tracts and also elsewhere in gardens (cultivated).

Distribution: India, Malay Peninsula, Myanmar and Sri Lanka. Cultivated in the Malay Island and elsewhere in the tropics.

Cryptocarya R. Brown, Prodr.: 402 (1810).

17. Cryptocarya amygdalina Nees in Wall., Pl. As. Rar. 2: 69 (1831). Hook. f., Fl. Brit. Ind. 5: 118 (1886). Cryptocarya floribunda Nees in Wall., Pl. As. Rar. 2: 69 (1831); Prain, Beng. Pl. 2: 671 (1903-reprint 1963); Heinig, List Chittagong: 56 (1925).

Local name: Bhuiya Gach.

A medium-sized to large tree up to $20 \mathrm{~m}$ high with spreading crown. Leaves 8-20 $\times$ 3-8 cm, broadly oblong, elliptic, elliptic-lanceolate or oblanceolate. Inflorescence terminal or axillary panicles, up to $15 \mathrm{~cm}$ long, pubescent, long peduncled and much branched. Fruit 2.0-2.5 × 0.9-1.2 cm, usually ribbed. Fl. \& Fr.: March-November. 
Occurrence in Bangladesh: Greater Chittagong and Sylhet forests (Hooker 1886, Prain 1903).

Distribution: Eastern Himalaya from Sikkim eastwards.

Dehaasia Blume, Rumphia 1: 161 (1835).

18. Dehaasia kurzii King ex Hook. f., Fl. Brit. Ind. 5: 125 (1886); Prain, Beng. Pl. 2: 672 (1903-reprint 1963); Heinig, List Chittagong: 56 (1925).

Local names: Modon-mosto, Bagraj (Chittagong, Chittagong Hill Tracts).

An evergreen tree. Leaves subverticillate, 15-25 × 3-6 cm, elliptic, acuminate or obtusely acuminate. Inflorescence panicles, long peduncled, very slender. Fruit a cylindrical to oblong berry, c $5 \mathrm{~cm}$ long. Fl. \& Fr.: August-December.

Occurrence in Bangladesh: Chittagong and Chittagong Hill Tracts (Heinig 1925).

Distribution: Andaman Island and Myanmar.

Endiandra R. Brown, Prodr.: 402 (1810).

19. Endiandra firma Nees in Wall., Pl. As. Rar. 2: 68 (1831). Hook. f., Fl. Brit. Ind. 5: 126 (1886); Kanjilal et al., Fl. Assam 4: 54 (1940-reprint 1982). Laurus firma Wall., Cat. no. 2597 (1830).

An evergreen tree, branches terete. Leaves alternate, 12-20 × 4-5 cm, elliptic-oblong, acuminate, glabrous. Inflorescence in short, axillary panicles, 2.5-5.0 cm long, branches spreading, puberulous. Fruit c $3 \mathrm{~cm}$ long, elliptic-ovoid, quite smooth, tip rounded. Fl. \& Fr.: October-December.

Occurrence in Bangladesh: Forests of Sylhet District (Hooker 1886, Alam 1988).

Distribution: India (Cachar).

Lindera Thumb., Nova Gen. Pl.: 64 (1783).

20. Lindera latifolia Hook. f., Fl. Brit. Ind. 5: 183 (1886). Brandis, Ind. Trees: 541 (1906); Heinig, List Chittagong: 57 (1925); Kanjilal et al., Fl. Assam 4: 95 (1940reprint 1982).

Local names: Shikoria, Shukaria, Shiori (Chittagong, Chittagong Hill Tracts).

An small to medium-sized evergreen tree, up to $20 \mathrm{~m}$ high. Leaves $10-20 \times 5-10 \mathrm{~cm}$, obovate, oblong or broadly oblanceolate and pinnately nerved. Inflorescence umbels, 10 12-flowered, very short peduncled. Fruit small, globose, 0.6-0.7 cm across. Fl. \& Fr.: February-October.

Occurrence in Bangladesh: Chittagong (Sitakund) and Chittagong Hill Tracts (Heinig 1925). 
Distribution: India (Meghalaya-Khasia hills).

21. Lindera melastomacea Benth., Gen. Plant. 3: 164 (1880). Hook. f., Fl. Brit. Ind. 5: 185 (1886); Brandis, Ind. Trees 541 (1906). Laurus cuspidata D. Don, Prodr. Fl. Nepal. 64 (1825).

A shrub or small tree, up to $10 \mathrm{~m}$ high. Leaves ascending, 3-10 $\times 1-3 \mathrm{~cm}$, obovate, oblanceolate or elliptic-oblong. Inflorescence umbels, globose, 3-5-flowered. Fruit c 0.8 cm long, ellipsoid, slightly apiculate. Fl. \& Fr.: February-November.

Occurrence in Bangladesh: Sylhet (Alam 1988).

Distribution: Eastern Himalaya and North-east India.

22. Lindera reticulata (Nees) Benth., Gen. Plant. 3: 164 (1880). Hook. f., Fl. Brit. Ind. 5: 183 (1886). Polyadenia reticulata Nees in Wall., Pl. As. Rar. 2: 61 (1831).

A small or medium-sized tree. Leaves $15-25 \times 3-8 \mathrm{~cm}$, obovate-oblong or oblanceolate to oblong. Inflorescence umbellate, very small, clustered, sessile. Fruit ellipsoid or oblong, $1.2 \mathrm{~cm}$ long, smooth, shortly pedicelled. Fl. \& Fr.: April-August.

Occurrence in Bangladesh: Sylhet (Hooker 1886).

Distribution: India.

Litsea Lamk., Dict. 3: 574 (1989).

23. Litsea angustifolia Wall. ex. Hook. f., Fl. Brit. Ind. 5: 169 (1886). Prain, Beng. Pl. 2: 676 (1903-reprint 1963); Heing, List Chittagong: 57 (1925); Kanjilal et al., Fl. Assam 4: 87 (1940-reprint 1982). Tetranthera saligna Nees in Wall., Pl. As. Rar. 2: 67 (1831).

Local names: Chhota Shiyal-Buka, Huria, Risapaing (Chittagong, Chittagong Hill Tracts).

A bushy evergreen shrub, young parts finely pubescent. Leaves alternate, 7.5-20.0 $\times$ 1.0-1.5, linear lanceolate. Umbellules solitary or fascicled. Fruit ellipsoid-oblong, 1.0-1.2 cm long, smooth. Fl. \& Fr.: March-September.

Occurrence in Bangladesh: Sylhet and Chittagong (Hooker 1886).

Distribution: India (Assam and Cachar).

24. Litsea clarkei Prain, Bengl. Pl. 2: 676 (1903). Heinig, List Chittagong: 50 (1925).

A small, evergreen tree. Leaves $7.5-12.5 \mathrm{~cm}$ long, alternate or subverticillate. Inflorescence umbels in solitary or binate racemes. Fruit unknown. Fl. \& Fr.: Not on record.

Occurrence in Bangladesh: Chittagong (Prain 1903).

Distribution: Endemic to Bangladesh (Khan et al. 2001). 
25. Litsea cubeba (Lour.) Pers., Syn. Pl. 2 (1): 4 (1806). Laurus cubeba Lour., Fl. Cochinch. 1: 252 (1970). Litsea citrata Blume, Bijdr.: 565 (1826); Hook. f., Fl. Brit. Ind. 5: 155 (1886). Litsea kingii Hook. f., Fl. Brit. Ind. 5: 156 (1886); Kanjilal et al., Fl. Assam 4: 81 (1940-reprint 1982).

A deciduous shrub or small tree. Leaves aromatic, alternate, $7.5-15 \times 2.5-4.0 \mathrm{~cm}$, lanceolate or narrowly ovate-lanceolate. Inflorescence in capitate umbels. Fruit 0.6-0.7 cm in diameter, globose. Fl. \& Fr.: November-July.

Occurrence in Bangladesh: Greater Sylhet (Alam 1988).

Distribution: Himalaya (Bhutan to Nepal), Indo-China, Myanmar and North-east India.

26. Litsea glutinosa (Lour.) Robinson in Philipp., J. Sci., Bot. 6: 321 (1911). Sinclair, Bull. Bot. Soc. Beng. 9 (2): 105 (1955). Sebifera glutinosa Lour., Fl. Cochinch.: 638 (1790). Litsea chinensis Lam., Encyc. Method III: 574 (1789); Datta \& Mitra., Bull. Bot. Soc. Beng. 7 (1\&2): 12 (1953). Litsea sebifera (Willd.) Pers., Syn. Pl. 2: 4 (1807); Hook. f., Fl. Brit. Ind. 5: 157 (1886); Prain, Beng. Pl. 2: 675 (1903-reprint 1963); Heinig, List Chittagong: 57 (1925).

Local names: Kukur-chita, Ratun, Garpur (Chittagong, Chittagong Hill Tracts).

A small to medium-sized, aromatic, evergreen tree, 5-18 m high. Leaves alternate, sub-terminal on the branches, very variable, 7.5-22.5 $\times 2.5-10.0 \mathrm{~cm}$, elliptic-oblong or ovate-lanceolate. Inflorescence 8-12-flowered umbellules. Fruit a spherical berry, 0.6-1.0 cm across. Fl. \& Fr.: April-January.

Occurrence in Bangladesh: Almost throughout the country.

Distribution: Australia, China, Malay Islands, India, Pakistan, Sikkim (lower Himalaya) and Sri Lanka.

27. Litsea laeta Wall. ex Nees in Wall., Pl. As. Rar. 2: 67 (1831). Hook. f., Fl. Brit. Ind. 5: 169 (1886). Tetranthera laeta Nees in Wall., Pl. As. Rar. 2: 67 (1831).

Local name: Bon-hoalu (Sylhet).

A small to medium-sized tree, up to $20 \mathrm{~m}$ high, with spreading branches. Leaves alternate, $10-30 \times 2-12 \mathrm{~cm}$, elliptic, lanceolate or narrowly oblong. Inflorescence umbels in axillary clusters. Fruit almost white, oblong-ovoid, $1.7 \mathrm{~cm}$ across. Fl. \& Fr.: November-April.

Occurrence in Bangladesh: Hill forests of greater Sylhet (Hooker 1886).

Distribution: Tropical and Eastern Himalaya, Bhutan, India and Sikkim. 
28. Litsea lancifolia (Roxb. ex Nees) Hook. f., Fl. Brit. Ind. 5: 159 (1886). Prain, Beng. Pl. 2: 676 (1903-reprint 1963); Heinig, List Chittagong: 57 (1925). Tetranthera lancifolia Roxb. ex Nees in Wall., Pl. As. Rar. 2: 65 (1831).

A shrub or small tree, up to $8 \mathrm{~m}$ high. Leaves opposite, sub-opposite or alternate, 7$15 \times 2-5 \mathrm{~cm}$. Inflorescence umbellate clusters. Fruit sub-globose or ellipsoid-oblong, 1$1.5 \mathrm{~cm}$ long and c $1.2 \mathrm{~cm}$ across, apiculate. Fl. \& Fr.: February-June.

Occurrence in Bangladesh: Greater Chittagong and Sylhet Districts (Hooker 1886, Heinig 1925).

Distribution: Tropical and Eastern Himalaya including Bhutan, India, Myanmar, Nepal and South China.

29. Litsea monopetala (Roxb.) Pers., Syn. Pl. 2: 4 (1807). Sinclair, Bull. Bot. Soc. Beng. 9 (2): 105 (1955). Tetranthera monopetala Roxb., Pl. Coromand. 2: 26. t. 148 (1798). Litsea polyantha Juss. in Ann. Mus. Hist. Nat. Paris 6: 211 (1805); Hook. f., Fl. Brit. Ind. 5: 162 (1886); Prain, Beng. Pl. 2: 676 (1903-reprint 1963); Heinig, List Chittagong: 57 (1925); Datta \& Mita., Bull. Bot. Soc. Beng. 7 (1 \& 2): 12 (1953).

Local names: Bara Kukur-chita, Huoria (Chittagong, Chittagong Hill Tracts, Sylhet).

A small to medium-sized, evergreen tree, usually 10-15 m high, with spreading crown. Leaves alternate, extremely variable, 7-20 × 3-12 cm, ovate-oblong, oblanceolate or elliptic-oblong. Inflorescence in pedunculate umbellate heads. Fruit globose to ellipsoid, 0.7-1.2 cm long, blackish when ripe. Fl. \& Fr.: March-November.

Occurrence in Bangladesh: Chittagong, Chittagong Hill Tracts (Heinig 1925) and Sylhet (Alam 1988).

Distribution: Bhutan, India, Malay Peninsula, Myanmar, Nepal and South-west China.

30. Litsea nitida Hook. f., Fl. Brit. Ind. 5: 174 (1886). Prain, Beng. Pl. 2: 676 (1903reprint 1963); Kanjilal, et al., Fl. Assam 4: 90 (1940-reprint 1982); Sinclair, Bull. Bot. Soc. Beng. 9 (2): 105 (1955). Tetranthera nitida Roxb. ex Nees, Wall. Pl. As. Rar. 2: 67 (1831).

A medium-sized tree. Leaves alternate, $10-25 \times 5-8 \mathrm{~cm}$, oblanceolate or obovateoblong. Inflorescence racemose. Fruit aromatic, ellipsoidal, 1-2 cm long. Fl. \& Fr.: November-April.

Occurrence in Bangladesh: Sylhet (Kanjilal et al. 1940).

Distribution: Bhutan, India, Myanmar and Nepal.

31. Litsea panamonja Hook. f., Fl. Brit. Ind. 5: 175 (1886). Prain, Beng. Pl. 2: 676 (1903-reprint 1963); Heinig, List Chittagong: 57 (1925). Tetranthera panamonja Nees in Wall., Pl. As. Rar. 2: 67 (1831). 
Local name: Panamonja (Chittagong).

A large tree, young shoot puberulous. Leaves 12-30 $\times 5-10 \mathrm{~cm}$, oblong or lanceolate. Male inflorescence umbels in long racemes. Female inflorescence umbels in short racemes. Fruit globose, broader than long. Fl. \& Fr.: March-May.

Occurrence in Bangladesh: Chittagong (Prain 1903) and Sylhet (Alam 1988).

Distribution: India (Assam), Malay Peninsula and Myanmar.

32. Litsea salicifolia (Roxb. ex Nees) Hook. f., Fl. Brit. Ind. 5: 167 (1886). Prain, Beng. Pl. 2: 676 (1903-reprint 1963); Heinig, List Chittagong: 57 (1925). Tetranthera salicifolia Roxb. ex Ness in wall., Pl. As. Rar. 2: 66 (1831).

Local names: Bara Shiyal, Digloti, Hiyal-buka, Pania-mula (Chittagong, Chittagong Hill Tracts, Sundarbans).

A small, evergreen tree or shrub up to $8 \mathrm{~m}$ high. Leaves alternate, very variable. Umbels 0.6-0.8 cm diameter, glabrous or hoary. Fruit 0.6-1.5 cm long, ellipsoid, obovoid. Fl. \& Fr.: February-May.

Occurrence in Bangladesh: Chittagong, Chittagong Hill Tracts (Heinig 1925) and Sylhet (Alam 1988).

Distribution: Myanmar, India, Nepal and Sikkim.

33. Litsea semecarpifolia (Wall.) Hook. f., Fl. Brit. Ind. 5: 165 (1886). Brandis, Ind. Trees: 537 (1906); Kanjilal et al., Fl. Assam 4: 86 (1940-reprint 1982). Tetranthera semecarpifolia Wall., Cat. n. 6345 A (1830).

A tree, 8-10 m high. Leaves alternate, 15-18 × 6-13 cm, obovate or elliptic-oblong. Inflorescence umbels, clusters of 6 flowers. Fruit globose, c $1.5 \mathrm{~cm}$ in diameter. Fl. \& Fr.: January-June.

Occurrence in Bangladesh: Recorded from the then East Bengal by Hooker (1886).

Distribution: India and Myanmar.

34. Litsea thomsonii Meissn. in. DC., Prodr. 15 (1): 183 (1964). Hook. f., Fl. Brit. Ind. 5: 170 (1886); Brandis, Ind. Trees: 538 (1906); Kanjilal et al., Fl. Assam 4: 89 (1940reprint 1982).

A large tree, branches stout. Leaves alternate, $12-25 \times 5-8 \mathrm{~cm}$, linear oblong or elliptic-lanceolate. Inflorescence axillary, umbels in short sub-racemous corymbs and in the leafless axils. Fruit globose, 1.5-2.0 cm across. Fl. \& Fr.: September-August.

Occurrence in Bangladesh: Sylhet (Hooker 1886, Alam 1988).

Distribution: India. 
Machilus C. G. D. Nees in Wall., Pl. As. Rar. 2: 61, 70 (1831).

35. Machilus fruticosa Kurz, Journ. As. Soc. Beng. 2: 101 (1873). Hook. f., Fl. Brit. Ind. 5: 140 (1886); Brandis, Ind. Trees: 531 (1906).

A glabrous shrub with velvety leaf-buds, branches stout, black. Leaves 6-15 cm long, ovate to ovate-oblong, linear or elliptic-oblong. Inflorescence axillary panicles, 7-20 cm long. Fruit small, globose, glabrous. Fl. \& Fr.: Not on record.

Occurrence in Bangladesh: Sitapahar of Rangamati District (Mia and Khan 1995).

Distribution: Myanmar.

Neolitsea (Benth.) Merr., Philip. J. Sci. 1 Suppl.: 56 (1906).

36. Neolitsea cassia (L.) Kosterm., J. Sci. Res. Indones. 1: 85 (1952). Laurus cassia L., Sp. Pl. 369 (1753). Neolitsea zeylanica (Nees) Merr. in Phillip. J. Sci. 1: Suppl. 57 (1906); Kanjilal et al., Fl. Assam 4: 92 (1940-reprint 1982). Litsea zeylanica C \& Fr. Nees in Amen. Bot. Bonn. Fasc. 1: 58, t. 5. (1823); Hook. f., Fl. Brit. Ind. 5: 178 (1886); Prain, Beng. Pl. 2: 676 (1903-reprint 1963); Heinig, List Chittagong: 57 (1925).

A small to medium-sized, evergreen tree, up to $20 \mathrm{~m}$ high. Leaves alternate, 7-15 $\times$ 3.5-5 cm, elliptic or elliptic-lanceolate. Inflorescence in 4-5-flowerd heads in almost sessile clusters. Fruit ovoid-oblong, 0.7-1.0 cm long. Fl. \& Fr.: October-April.

Occurrence in Bangladesh: Chittagong, Chittagong Hill Tracts and Sylhet (Hooker 1886, Heinig 1925).

Distribution: Eastern Himalaya, Bhutan, Indo-Malesia and Myanmar.

Persea P. Mill., Gard. Dic. Abr. ed. 4 (1754).

37. Persea americana P. Mill., Gard. Dict. ed. 8 (1768).

Local name: Avocado. English name: Avocado Pear.

A tree usually $15 \mathrm{~m}$ high with spreading crown. Leaves 8-18 $\times 4-6 \mathrm{~cm}$, oblong to elliptic. Inflorescence compact panicles, terminal on branchlets. Fruit a berry, obovoid, $10 \times 8 \mathrm{~cm}$. Fl. \& Fr.: March-May.

Occurrence in Bangladesh: Mirpur National Botanic Garden, Dhaka (Dey 2006).

Distribution: Tropical America, widely cultivated in tropics.

38. Persea bombycina (King ex Hook. f.) Kosterm., in Reinwardtia 6: 191 (1962). Machilus bombycina King ex Hook. f., Fl. Brit. Ind. 5: 861 (1890); Prain, Beng. Pl. 2: 674 (1903-reprint 1963); Heinig, List Chittagong: 56 (1925); Kanjilal et al., Fl. Assam 4: 66 (1940-reprint 1982). 
A medium-sized tree up to $20 \mathrm{~m}$ high. Leaves alternate, 6-13 $\times 2-5 \mathrm{~cm}$, ellipticlanceolate to ovate-lanceolate. Inflorescence sub-terminal panicles, up to $10 \mathrm{~cm}$ long, spreading, sparsely pubescent. Fruit globose, 0.5-0.8 cm across. Fl. \& Fr.: DecemberMay.

Occurrence in Bangladesh: Greater Chittagong and Sylhet Districts (Prain 1903, Alam 1988).

Distribution: Lower Himalaya including India (Assam), Myanmar and Nepal.

39. Persea gamblei (King ex Hook. f.) Kosterm., Reinwardtia 6: 192 (1962). Machilus gamblei King ex Hook. f., Fl. Brit. Ind. 5: 138 (1886); Prain, Beng. Pl. 2: 673 (1903reprint 1963); Brandis, Ind. Trees: 531 (1906); Kanjilal et al., Fl. Assam 4: 67 (1940).

A medium-sized to large tree, up to $20 \mathrm{~m}$ high. Leaves $6-15 \times 2-6 \mathrm{~cm}$, variable in shape, obovate-oblong, oblanceolate or lanceolate to elliptic. Inflorescence in lax, fewflowered, pubescent panicles, 3-7 cm long. Fruit 0.7-1.0 cm across, black and primrose when ripe. Fl. \& Fr.: January-July.

Occurrence in Bangladesh: North Bengal (Prain 1903).

Distribution: Bhutan, North-East India and Nepal.

40. Persea odoratissima (Nees) Kosterm., in J. Sci. Res. Indonesia 1: 116 (1952). Machilus odoratissima Nees in Wall., Pl. As. Rar. 2: 70 (1831); Hook. f., Fl. Brit. Ind. 5: 139 (1886); Brandis, Ind. Trees: 530 (1906); Kanjilal et al., Fl. Assam 4: 64 (1940-reprint 1982).

A medium-sized tree, up to $15 \mathrm{~m}$ high. Leaves crowded at the ends of branches, 7-18 $\times$ 2-5 cm, very variable in shape, oblanceolate, oblong-lanceolate, elliptic-oblong. Inflorescence sub-terminal panicles, up to $13 \mathrm{~cm}$ long and many flowered. Fruit 1.2-1.6 cm long, oblong or ellipsoid, purple and primrose when ripe. Fl. \& Fr.: March-June.

Occurrence in Bangladesh: Sylhet (Kanjilal et al. 1940).

Distribution: Subtropical and Temperate Himalaya, Bhutan, China, India, Malaysia and Myanmar.

41. Persea owdeni (Parker) Kosterm., Lauraceae in Pulle, Fl. Suriname 2 (1): 244-337 (1936). Alseodaphne owdeni Parker in Indian Forester 50: 365 (1924); Kanjilal et al., Fl. Assam 4: 62 (1940-reprint 1982).

Local names: Jatisundi, Maricha Sundi, Tilsundi (Sylhet).

A large tree, branches glabrous. Leaves alternate, 7-15 × 2.5-4 cm, lanceolate. Inflorescence panicles, lax, from beneath of the crowded leaves. Fruit a drupe, 0.2-1.0 cm long, bluish-black when ripe, ellipsoid. Fl. \& Fr.: May-September. 
Occurrence in Bangladesh: Sylhet (Alam 1988).

Distribution: India.

42. Persea villosa (Roxb.) Kosterm., Reinwardtia 6 : 194 (1962). Laurus villosa Roxb. Fl. Ind. 2: 310 (1824). Phoebe villosa (Roxb.) Wight, Ic. Pl. Ind. Or. 5: 11, t. 1822 (1852). Machilus villosa (Roxb.) Hook. f., Fl. Brit. Ind. 5: 860 (1890); Prain, Beng. Pl. 2: 673 (1903-reprint 1963); Heinig, List Chittagong: 56 (1925).

A medium to large tree, up to $25 \mathrm{~m}$ high with spreading crown. Leaves alternate, 7$17 \times 2.5-4.0 \mathrm{~cm}$, elliptic to lanceolate. Inflorescence in panicles, up to $18 \mathrm{~cm}$ long, subterminal. Fruit a berry, globose, 0.5-0.8 cm across with reflexed perianth. Fl. \& Fr.: December-May.

Occurrence in Bangladesh: Chittagong and Sylhet (Hooker 1886).

Distribution: North-east India, Myanmar, Nepal to Sikkim.

Phoebe C.G.D. Nees, Syst. Laurin.: 98 (1836).

43. Phoebe attenuata (Nees) Nees, Syst. Laur.: 104 (1836). Hook. f., Fl. Brit. Ind. 5: 143 (1886). Ocotea attenuata Nees in Wall., Pl. As. Rar. 2: 71 (1831).

Local name: Bonsum (Sylhet).

A large tree, often reaching 25-30 m high. Leaves alternate, crowded at the ends of branchlets, $12-25 \times 4-8 \mathrm{~cm}$, oblong or oblanceolate. Inflorescence spreading panicles, pedunculate, peduncles stout, 7.5-15.0 cm long. Fruit c $1.2 \mathrm{~cm}$ long, narrowly ellipsoid. Fl. \& Fr.: March-October.

Occurrence in Bangladesh: Sylhet (Gamble 1922, Alam 1988).

Distribution: Bhutan, India (Assam), Nepal and Sikkim.

44. Phoebe cathia (Buch.-Ham. ex D. Don) Kosterm., Nat. Hist. Bull. Siam S. 25: 44 (1974). Cinnamomum cathia Buch.-Ham. ex D. Don, Prodr. Fl. Nep. 66 (1825). Ocotea paniculata Nees in Wall., Pl. As. Rar. 2: 71 (1831); 3: 32 (1832). Phoebe paniculata (Nees) Nees, Syst. Laur.: 105 (1836); Hook. f., Fl. Brit. Ind. 5: 142 (1886); Prain, Beng. Pl. 2: 674 (1903-reprint 1963); Heinig, List Chittagong: 56 (1925).

A tall tree, up to $2 \mathrm{~m}$ high. Leaves 7-22 $\times 2-20 \mathrm{~cm}$, crowded at the ends of twings, elliptic, oblong-obovate or oblong-elliptic. Inflorescence panicles, 6-10 cm long. Fruit a berry, c $1.0 \times 0.8 \mathrm{~cm}$, ovoid, reticulately rugose, blackish. Fl. \& Fr.: March-November.

Occurrence in Bangladesh: Chittagong (Hooker 1886, Prain 1903).

Distribution: India, Myanmar and Nepal. 
45. Phoebe lanceolata (Nees) Nees, Syst. Laur.: 109 (1836). Hook. f., Fl. Brit. Ind. 5: 141 (1886); Prain, Beng. Pl. 2: 674 (1903-reprint 1963); Heinig, List Chittagong: 56 (1925). Ocotea lanceolata Nees in Wall., Pl. As. Rar. 2: 71 (1831).

Local names: Chaongri, Dulia (Chittagong, Chittagong Hill Tracts).

A small to medium-sized evergreen tree, up to $10 \mathrm{~m}$ high, with spreading branches. Leaves alternate, crowded towards the apex, 8-25 $\times 2-6 \mathrm{~cm}$, lanceolate or oblonglanceolate or elliptic-lanceolate. Inflorescence axillary corymbiform panicles, longpeduncled, sometimes 10-15 cm long. Fruit a berry, 0.7-1.2 cm long, black, ovoid or ellipsoidal. Fl. \& Fr.: April-November.

Occurrence in Bangladesh: Chittagong, Chittagong Hill Tracts (Heinig 1925) and Sylhet (Alam 1988).

Distribution: Bhutan, India, Myanmar and Nepal.

46. Phoebe pallida (Nees) Nees, Syst. Laur.: 112 (1836). Hook. f., Fl. Brit. Ind. 5: 142 (1886). Ocotea pallida Nees in Wall., Pl. As. Rar. 2: 71 (1831).

A small to medium-sized tree, young parts of shoots very finely puberulous. Leaves alternate, 7-20 × 2-4 cm, oblanceolate or elliptic-lanceolate, minutely pubescent when young, glabrous when mature. Inflorescence axillary in lax panicles, peduncles up to 13 cm long, puberulous. Fruit c 1 cm long, ellipsoid. Fl. \& Fr.: June-January.

Occurrence in Bangladesh: Sylhet (Alam 1988).

Distribution: North India, Myanmar, Nepal and Sikkim.

\section{References}

Alam, M.K. 1988. Annotated checklist of the woody Flora of Sylhet Forests. Forest Research Institute, Chittagong, pp. 1-153.

Brandis, D. 1874. The Forest Flora of North-West and Central India. Indian reprint 1972. Bishen Singh Mahendra Pal Singh, Dehra Dun, India, pp. 1-608.

Brandis, T.D. 1906. Indian Trees. Indian reprint 1978. Periodic Experts Book Agency, Delhi, India, pp. 1-767.

Cowan, A.M. and Cowan, J.N. 1929. The Trees of Northern Bengal. Bengal Secretariat Book Depot, Calcutta, pp. 1-178.

Datta, R.M. and Mitra, J.N. 1953. Common Plants in and Around Dacca. Bull. Bot. Soc. Beng. 7(1-2):1-110.

Dey, T.K. 2006. Bangladesher Praojanio Gachgachra (Useful Plants of Bangladesh). The Ad. Communication, Anderkila, Chittagong, p. 1-987.

Farr, E.R., Leussink, J.A. and Stafleu, F.A. (eds). 1979. Index Nominum Genericorum (Plantarum). Vols 1-3. Bohn, Scheltema and Holkema, Utrecht Dr. W. Junk B.V., Publishers, The Hague, The Netherlands, pp. 1-1896.

Gamble, J.S. 1922. A Manual of Indian Timbers. Indian reprint 1984. Bishen Singh Mahendra Pal Singh, Dehra Dun, India, pp. 1-868.

Hara, H., Chater, A.O. and Williams, L.H.J. 1982. An enumeration of the Flowering Plants of Nepal 3. Trustees of British Museum (Natural History), London, pp. 1-226. 
Heinig, R.L. 1925. List of plants of Chittagong Collectorate and Hill Tracts. The Bengal Government Branch Press, Darjeeling, India, pp. 1-89.

Heywood, V.H. 1993. Flowering Plants of the World. Oxford University Press, London, pp. 1-336.

Hooker, J.D. 1886, 1890. Lauraceae. Flora of British India 5. Indian reprint 1973. Bishen Singh Mahendra Pal Singh, Dehra Dun, India, pp. 1-910.

Huq, A.M. and Khan, M.S. 1984. A preliminary taxonomic report on the angiospermic flora of Moheskhali Island-1 (Dicotyledons). Dhaka Univ. Studies. Part B 32(2): 19-31.

Kanjilal, U.N., Kanjilal, P.C., De, R.N. and Das, A. 1940. Flora of Assam 4. Indian reprint 1982. A Von Book Company, Delhi, India, pp. 1-337.

Khan, M.S., Rahman, M.M., Huq, A.M., Mia, M.M.K. and Hassan, M.A. 1994. Assessment of biodiversity of Teknaf Game Reserve in Bangladesh focussing on economically and ecologically important plant species. Bangladesh J. Plant Taxon. 1(1): 21-33.

Khan, M.S. and Huq. A.M. 2001. The vascular flora of Chunati Wildlife Sanctuary in South Chittagong, Bangladesh. Bangladesh J. Plant Taxon. 8(1): 47-64.

Khan, M.S., Rahman, M.M. and Ali, M.A. (eds). 2001. Red Data Book of Vascular Plants of Bangladesh. Bangladesh National Herbarium, Dhaka, pp. 1-179.

Kostermans, A.J.G.H. 1978. Lauraceae in Flora of West Pakistan. No. 118. Rijksherbarium, Leiden, Netherlands, pp. 1-13.

Mia, M.M.K. and Khan, B. 1995. First list of angiospermic taxa of Bangladesh not included in Hooker's 'Flora of British India' and Prain's 'Bengal Plants'. Bangladesh J. Plant. Taxon. 2(1\&2): 25-45.

Prain. D. 1903. Bengal Plants 2. Indian reprint 1963. Botanical Survey of India, Catcutta, pp. 491-1013.

Rahman, M.A. and Uddin, S.B. 1997. Angiospermic flora of Sitakundu in Chittagong, Bangladesh. Bangladesh J. Plant Taxon. 4(1): 17-36.

Rahman, M.O. 2004a. Second list of angiospermic taxa of Bangladesh not included in Hooker's 'Flora of British India' and Prain's 'Bengal Plants': Series I. Bangladesh J. Plant Taxon. 11(1): 77-82.

Rahman, M.O. 2004b. Second list of angiospermic taxa of Bangladesh not included in Hooker's 'Flora of British India' and Parin's 'Bengal Plant's: Series II. Bangladesh J. Plant Taxon. 11(2): 49-56.

Rahman, M.O. and Hassan, M.A. 1995. Angiospermic flora of Bhawal National Park, Gazipur, Bangladesh. Bangladesh J. Plant Taxon. 2(1 \& 2): 47-79.

Raizada, M.B. 1941. On the Flora of Chittagong. Indian Forester 67(5): 245-254.

Rashid, M.H., Rahman, E. and Rahman, M.A. 2000. Additions to the angiospermic flora of the Moheskhali Island, Cox's Bazar, Bangladesh. Bangladesh J. Plant Taxon. 7(1): 43-63.

Sinclair, J. 1955. Flora of Cox's Bazar, East Pakistan, Bull. Bot. Soc. Bengal. 9(2): 84-116.

Uddin, S.B. and Rahman, M.A. 1999. Angiospermic flora of Himchari National Park, Cox’s Bazar, Bangladesh. Bangladesh J. Plant Taxon. 6(1): 31-64.

Uddin, S.N., Khan, M.S., Hassan, M.A. and Alam, M.K. 1998. An annotated checklist of angiospermic flora of Sita Pahar at Kaptai in Bangladesh. Bangladesh J. Plant Taxon. 5(1): 13-46.

(Manuscript received on 4 October 2007; revised on 10 November 2007) 